Perspectiva Geográfica

ISSN 0123-3769

Vol. 15/2010; pp. 61-84

\title{
Tránsito de la sociedad rural agrícola a la sociedad urbana industrial en Bogotá durante las primeras décadas del siglo $\mathrm{xx}$
}

Transition from a rural agricultural society to an urban Industrial society in Bogota during the first decades of the twentieth century

Luis Gabriel Duquino Rojas*

\section{Resumen}

El artículo expone resultados parciales del trabajo de investigación titulado "Sustentabilidad ambiental en Bogotá. Evolucióndel modo de producción, la formación económica social y el espacio urbano en Bogotá (1920 2010)", realizado por el autor para optar al título de Magister en Geografía con énfasis en ordenamiento territorial. El trabajo se sustenta teórica y metodológicamente en el "materialismo histórico geográfico" (Harvey, 2003) y en el discurso de la "sustentabilidad ambiental" (Leff, 2002). Se realizó una revisión de las intensas relaciones entre los cambios ocurridos en el modo de producción (del capitalismo comercial fundado en el modelo rural/agrícola al capitalismo industrial manifiesto en el modelo urbano/ industrial), las formaciones sociales y la expresión espacial urbana de estos fenómenos. El artículo contiene el mencionado análisis en el territorio de Bogotá para las primeras décadas del siglo xx.

Palabras clave: Barrios obreros, Industrialización, Lucha de clases, Modernización, Modo de producción, urbanización.

* Magíster en Geografía. Funcionario de la Secretaría Distrital de Planeación de Bogotá. Iduquino@sdp.gov.co; Igdrojas@yahoo.com.mx 


\begin{abstract}
This paper presents partial results of the research entitled "Environmental Sustainability in Bogota. Evolution of mode of production, social economic formation and urban space in Bogotá (1920 2010)", developed by the author in order to apply and obtain the title of Master in Geography with an emphasis on land use. This work is supported theoretically and methodologically in the "historical geographical materialism" (Harvey, 2003) and the discourse of "environmental sustainability" (Leff, 2002). A review of the intense relationships between changes in the mode of production (from commercial capitalism based on the rural/agricultural model to industrial capitalism manifest in the urban/industrial model), social formations and the urban spatial expression of these phenomena. The article contains the above analysis in the territory of Bogota through the first decades of the twentieth century.
\end{abstract}

Key words: Class districts, Industrialization, Class struggle, Modernization, Production mode, Urbanization.

62 Luis Gabriel DuQuino Rojas 


\section{Introducción}

Durante los años finales del siglo XIX y los iniciales del siglo xx, el país, y Bogotá en particular, comenzaron a experimentar transformaciones que llevaron al tránsito de una fase económica con énfasis en la actividad agrícola y minera de corte extractivista, orientada a la exportación y cuya forma de ocupación primordial fue la rural, a una con énfasis en la actividad industrial, orientada a la satisfacción del mercado interno y cuyo espacio paradigmático fue el urbano. Dicha transformación se encuentra estrechamente relacionada con cambios en las ideologías políticas hegemónicas, con la recomposición de las élites locales y la aparición de nuevos actores sociales de base, que bajo las nuevas circunstancias socioeconómicas fueron construyendo una expresión espacial en el territorio de acuerdo con sus posibilidades de intervención y participación en las estructuras de poder. A continuación se espera dar cuenta del momento histórico en que se manifestó con más fuerza la transformación política, social, económica y espacial: las primeras décadas del siglo $\mathrm{xx}$.

\section{Transformación de la espacia- lidad en Bogotá. De pueblo grande a ciudad incipiente}

\section{Caracterización}

Bogotá se localiza en una unidad geomorfológica de piedemonte (1), sobre una planicie central de la cordillera Oriental de los andes colombianos. En los años veinte del siglo $\mathrm{xx}$, la configuración de la ciudad se desarrolla, principalmente, en el eje norte-sur, colindando hacia el occidente con un sector plano, antiguamente lago (2), que presenta un rico sistema hídrico de ríos, quebradas y escorrentías que bajan de la montaña, y que se acompañan de humedales y chucuas que sirven de sistema natural de drenaje. El sector occidental de este territorio se encuentra delimitado por el río Bogotá (3), que discurre paralelo a las montañas orientales (4) (Figura 1).

Sobre este territorio, el modelo de ocupación en la Colonia, caracterizado morfológicamente por la retícula espacial del damero, la presencia de una plaza mayor, la zonificación a partir de parroquias, la escasez de espacios públicos, grandes casas con solar, huerta y zaguán, y la monumentalidad reservada para los edificios religiosos (Mejía, 1998), se mantuvo más allá del periodo colonial y caracterizó la ciudad hasta bien entrado el siglo xx. La lenta transformación de Bogotá hacia una precaria e incompleta configuración moderna va a llevarse a cabo en el periodo histórico comprendido por las primeras cinco décadas del siglo $\mathrm{xx}$, en parte como un proceso que se caracteriza más por su espontaneidad que por su planificación; esto sin demeritar la existencia de modelos y propuestas de planificación que de algún modo 


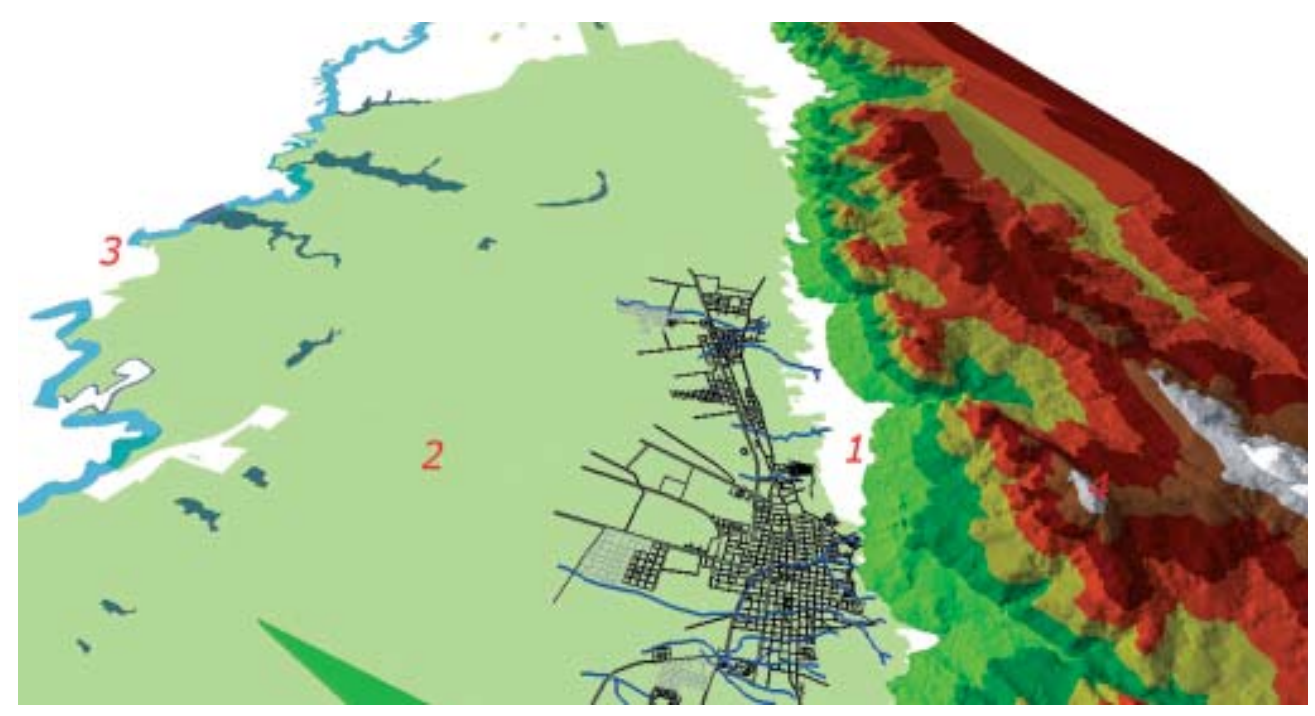

Figura 1. Conformación del espacio geográfico de Bogotá

Fuente: Coberturas, Base de Datos Geográfica SDP, CD de Bogotá Museo de Desarrollo Urbano. Montaje del autor.

intentaron orientar los cambios en aras de alcanzar una modernización urbana completa (Del Castillo, 2003).

Paulatinamente, este proceso de modernización se fue expresando en aspectos como la construcción de infraestructuras viales y de servicios públicos; la implantación de edificaciones con nuevos estilos arquitectónicos -fruto de la diversificación de los usos del suelo y de los servicios que comienza a prestar el espacio urbano, de la densificación de la ciudad y de su crecimiento demográfico sostenido-, y la aparición del aparato productivo industrial moderno (de corte fordista), que trae consigo una estructura edilicia a su servicio, y la vivienda obrera, como categoría residencial distinta a los alojamientos de la ciudad colonial.

En la siguiente cita se pueden observar algunas de las características anteriormente descritas, las cuales reflejan el estancamiento, en términos de evolución urbana, en el que se encontraba inmersa la ciudad en las primeras décadas del siglo xx, como consecuencia, entre otros, de los procesos socioeconómicos que vivía el país.

La población exacta de Bogotá es tan incierta como la del país. El último censo, en 1884, le dio 95.761 habitantes. Desde entonces Bogotá ha sufrido menos que el resto del país los efectos ruinosos de la guerra civil $\mathrm{y}$, así, es seguro asumir que su

64 Luis Gabriel Duquino Rojas 
población actual pasa de 100.000 habitantes, que representan las razas autóctonas y muchos de los cruces intermedios que se ven en las calles, aunque la sangre negra es escasa, y elemento predominante es el blanco o mestizo. Vista la ciudad desde lo alto da la impresión de un nivel homogéneo de techos rojizos. Las torres y cúpulas de las iglesias y los grupos ocasionales de casas que se elevan sobre el nivel general, de una o dos plantas, no distraen la vista. La atmósfera es tan clara y sin contaminaciones de humo que una toalla colgada, abajo en una azotea, puede distinguirse a simple vista desde la capilla de Guadalupe a 2.300 pies de altura. Y lejos, hacia el norte y separado de la ciudad por milla y media de quintas, los tejados rojos del gran suburbio de Chapinero se destacan en medio de la planicie verde (F. Loraine Petre, 1906; citado por Martínez, 1978: 125).

Las transformaciones espaciales que se desarrollaron en la ciudad van a dar cuenta de los cambios económicos y sociales que se han venido analizando. Los vínculos entre la evolución material del proceso histórico, manifestado en el aparato productivo, las comunidades que lo constituyen y la espacialidad que se construye para escenificar y experimentar estos procesos, son fuertes e indisolubles. La dimensión espacial y geográfica de los procesos económicos y sociales propios del tránsito hacia la implantación de un modelo capitalista industrial tiene en la expresión territorial una manifestación directa tanto de los objetivos de dominio e imposición, como del imaginario construido alrededor de los deseos y sueños de los individuos y de las colectividades: de un lado, los que con más facilidad se imponen, los de las clases sociales más influyentes, y de otro lado, los que se construyen desde la marginalidad de las clases rezagadas por la evolución de los procesos mencionados. La idea de territorio ${ }^{1}$ sugiere un constructo en el cual convergen las fuerzas socioeconómicas en la implantación de su propio poder para gestionar el espacio habitable. Son las lógicas impuestas, las concertadas y las marginales las que van delineando el proceso de construcción de la realidad territorial de la naciente ciudad. De este modo, la imposición de nuevas dinámicas desde el aparato de dominio capitalista, que abarcan las escalas de la intervención internacional -cifradas principalmente en la influencia hegemónica de EE.UU., para el caso de estudio-, hasta la actuación de las élites locales en los procesos de reacomodamiento del poder político y económico, van constituyendo una espacialidad propia, disímil de los ancestrales ordenamientos subyugados a la producción colonial de la hacienda. Se puede observar cómo la nueva estructura de dominio (externa y local) tendrá algún

\footnotetext{
1 Para esta investigación, el territorio se define como una construcción social en cuyo proceso de concreción se desarrolla una actividad espacial de los actores sociales que lo construyen, la cual es diferencial y desigual, generando características de movilidad, mutación y desequilibrio. De igual forma, el territorio se soporta en la posibilidad de ser expresado como un espacio de poder, de gestión y de dominio del Estado, a través del empoderamiento que le otorga la sociedad que compone dicho Estado (Montañez y Delgado, 1998).
} 
nivel de gestión en la construcción de la realidad espacial, dejando los aspectos de dicha realidad que les resulten menos atrayentes para el proceso de acumulación capitalista a la indeterminación de la acomodación propia de los flujos constitutivos.

Precisamente, uno de los escenarios en donde se va a representar la contradicción dialéctica de las fuerzas sociales que representan la lucha entre lo rural y lo urbano es la ciudad que transita el proceso de consolidación espacial a partir de la disolución de las haciendas y las quintas ${ }^{2}$ en la sabana de Bogotá. En la medida en que las presiones por suelo urbano generaron una demanda sólida e hicieron del proceso urbanizador una actividad lucrativa, los hacendados de los emplazamientos más cercanos a los límites urbanos de la época comenzaron el proceso de parcelación de sus terrenos, cediendo al ánimo especulador que iba creciendo. Dichos hacendados representaban a las pocas manos en las cuales tradicionalmente se concentraba la propiedad de la tierra en el país y, por consiguiente, en la sabana, tal como dan cuenta de ello Vergara y Velasco en 1890: "la Sabana pertenece a 30 dueños, alguno de los cuales posee hasta 5.000 Hectáreas de los mejores terrenos. Otra finca que ocupa llanura y cordillera mide 16.000 Hectáreas" (citado en Mejía, 1998: 43).
Las haciendas y quintas más relevantes (figura 2) en el proceso de conformación de la realidad urbana de Bogotá son las siguientes (Del Castillo, 2003): Hacienda Chapinero (1), de 2.320 ha, que fue la primera en parcelarse, de propiedad de José Antonio Sánchez, quien además fue dueño de las haciendas Tibabuyes (2), Punta de Suba (3), Juan Amarillo (4) y Tintal (5); Hacienda El Salitre (6), de 1.400 ha, propiedad de Sebastián Herrera hasta 1936, cuando es adquirida por José Joaquín Vargas, propietario también de la hacienda El Ejido (7); las quintas La Soledad y La Merced (8), propiedad de José María Malo Blanco; la quinta La Magdalena (9), por donde discurrían la quebrada Tisquesusa y el río Arzobispo, en su parte baja, propiedad de la familia Espinosa. La quinta del río Arzobispo (10), donde hoy se localizan el Parque Nacional, el barrio La Merced y el Colegio San Bartolomé, propiedad de la familia Maldonado. A principios de siglo, Pepe Sierra adquiere las Haciendas Chicó Manrique, Chicó Saiz (11) y Santa Bárbara (12), al norte de la ciudad. En el sur de la ciudad también se encontraban grandes haciendas, entre las que se destacan La Milagrosa (13), Hacienda de Ajos Tunjuelo (14), Molino de Chingaza (15), San Vicente (16) y Llano de Mesa (17).

2 "Quinta es la hacienda de labor en el campo con su caserío. Díjose así porque el arrendador della da al señor por concierto la quinta parte de lo que coge de frutos. Lo mismo significa quintería y quintero es el tal arrendador" (Monlau, Pedro, Diccionario Etimológico de la Lengua Castellana, 1856, citado en Carrasquilla, 1989, 12). 


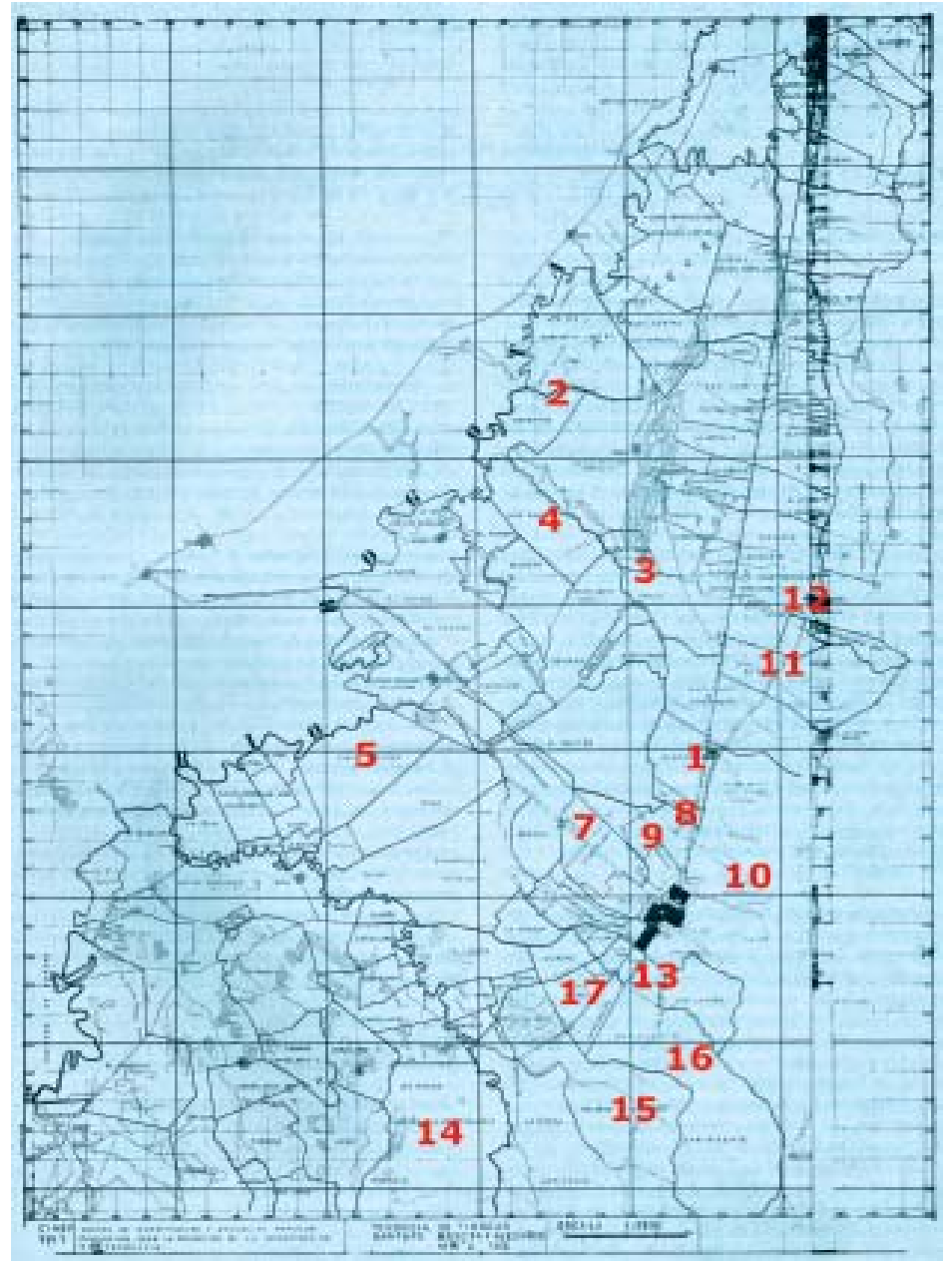

Figura 2. Quintas y haciendas en la periferia de Bogotá 1875 a 1900

Fuente: Carrasquilla, 1989, p. 193.

\section{Industrialización y barrios obreros}

Uno de los principales elementos del proceso de industrialización fordista naciente tiene relación directa con la dinámica del modelo de ocupación urbana pergeñado en la triada modernidadindustrialización-urbanización, que explicita la vinculación de los nuevos modos de producción a la configuración espacial urbana y a la relevancia del espacio de la ciudad, en la cual se materializa una sociedad moderna, industrial y urbana; moderna, desde la ideología, el deseo y el imaginario; industrial, desde las nuevas lógicas de reproducción del capital, y urbana, desde 
la conglomeración de las poblaciones en los espacios de la ciudad, bien sea por el desplazamiento forzado fruto de la violencia del campo colombiano, o de la falta de condiciones de subsistencia, en contraposición a las oportunidades en términos de empleo y servicios que se empiezan a constituir en las ciudades florecientes. La aparición de nuevas industrias y empresas manufactureras (Figura 3), y el fortalecimiento de las existentes en Bogotá, generaron nuevas lógicas espaciales fundamentadas en el espíritu funcional de estos elementos.

Para principios de la década de los veinte, "En Bogotá y Cundinamarca se había instalado la Cervecería Bavaria, una fábrica de cemento, dos fábricas de tejidos, la fábrica nacional de fósforos, una fábrica de calzado y pequeños establecimientos en los que se fabricaban jabones, velas y pastas alimenticias" (Ocampo, 2007: 210). Esta incipiente transformación de los sistemas de producción implantados en el espacio geográfico de la ciudad trajo una serie de dinámicas nuevas e incentivó la consolidación de otras, asociadas a las actividades de manufactura artesanal y de comercio, que ya existían en el siglo XIX.

El aparato industrial que se va constituyendo necesita que la mano de obra tenga fácil acceso a los incipientes polos industriales, lo que generó la organización de barrios obreros. En el desarrollo de estas nuevas formas de ocupación espacial se presentaron diversas modalidades de intervención, planificada o informal, además de variaciones en la organización de la gestión que acompaña los procesos de surgimiento de los barrios obreros: gestión pública, cooperativa, privada $^{3} \mathrm{o}$ simplemente ilegal (Amézquita, 2007). Con posterioridad, en la década de los treinta $^{4}$, se comienza a fundamentar la institucionalidad pública que se encargará del manejo y gestión de la temática de vivienda obrera.

En los años veinte, el término "barrio obrero" está asociado en el imaginario público a una vivienda precaria, periférica, de condiciones insalubres, que aloja habitantes con "hábitos morales" degradados; se fija así, de manera peyorativa, una distancia respecto a los barrios residenciales de la burguesía de la ciudad. Por tanto, la definición misma del término, hasta las consideraciones más técnicas, asociadas a la localización y diseño de estos espacios, tienen originalmente un sesgo de marginalidad y segregación. En medio del discurso higienista que se afianzó durante las primeras décadas del siglo $\mathrm{xx}$, bajo el cual se asumió la problemática de la

\footnotetext{
3 En términos de la gestión privada cabe destacar el papel de los industriales que invirtieron en el negocio de ofrecer vivienda para sus propios trabajadores, como es el caso del barrio Unión Obrera, hoy La Perseverancia, cuya construcción fue impulsada por el propietario de Bavaria, Leo Koop.

4 En 1936 se funda la "Oficina de Planos Obreros", primer ente público de la ciudad encargado de la vivienda obrera (Amézquita, 2007).
}

68 Luis Gabriel DuQuino Rojas 
vivienda obrera, se dio inicio a una crítica profunda a las condiciones de estos espacios urbanos, llegando a otorgar gran parte de la responsabilidad de la situación de la insalubridad de la ciudad a estos espacios y sus habitantes. En referencia al sector del Paseo Bolívar, uno de los barrios obreros de la época ubicado en el sector oriental de la ciudad, entre la plaza de Egipto y la parte alta del Parque de la Independencia, se señalaba lo siguiente:

...una "corona infectante" que la domina, viciándola con más eficacia que si la caja de Pandora se hubiera abierto por aquellos lados. Esta zona arroja en verano sobre la ciudad, a favor del viento, el polvo impregnado de gérmenes de toda especie, y en invierno, con el agua que de allí desciende en formidables avenidas que van a depositarlos en todas las calles, carreras, plazas y casas que en la parte baja inundan (Registro Municipal de Higiene N. ${ }^{\circ} 11,1915$, citado en Colón, 2007: 106).

Acompañando el discurso higienista se observa, de forma paralela, cómo se incluye un discurso moral, en el cual se promulga la posibilidad de modificar los comportamientos de las clases sociales que habitan estos espacios a través de la configuración misma de su vivienda.

...si la casa está mal dispuesta, si carece de aire y luz, el trabajador permanece en ella lo menos posible y prefiere las funestas diversiones de la taberna. Cada día se desprende más de su mujer y de sus hijos, quienes quedan abandonados a la miseria y a ejemplos perniciosos. Las pasiones de las colectividades sin freno, excitadas por el abuso del alcohol, arrastran al obrero a falsos conceptos de venganza y de odio, con el deseo de quiméricas reivindicaciones (Vergara y Vergara, 1919; citado en Colón, 2007: 109).

En la caracterización inicial que se hace de las áreas de alojamiento obrero existe una asociación básica de estas zonas y sus habitantes con condiciones físicas y morales malsanas, que atentan contra la vida sana y las buenas costumbres de la ciudad burguesa. Espacialmente, los barrios obreros de las primeras décadas del siglo $\mathrm{xx}$ se localizaron sobre las periferias del desarrollo de la ciudad central, continua y compacta. De la siguiente descripción que se hace de estos sectores de Bogotá en la época se puede inferir la distribución de estos espacios y su relación con otros sectores funcionales de la ciudad (figura 3):

Unión obrera (hoy conocido como la Perseverancia); Bavaria, contiguo al anterior, abarcaba las cuadras comprendidas entre las calles 29 y 32 , de la carrera 5 al oriente; Antonio Ricaurte, situado entre las calles 7 y 12 y las carreras 23 y 27; Uribe Uribe, situado al occidente de Chapinero, de la carrera 18 abajo entre las calles 65 y 68; San Francisco Javier, situado al sur de la ciudad. El sector de la ciudad que agrupaba el mayor número de "barrios obreros" era el del Paseo Bolívar, comprendido entre el Parque de la Independencia y la Plaza del barrio Egipto, al oriente e incluía los siguientes barrios: Barrio San Ignacio de Loyola 1, situado entre las calles 26 y 27 , de la carrera 4 al oriente; 
San Ignacio de Loyola 2, situado al lado del anterior; San Luis, situado arriba del Paseo Bolívar, frente a la calle 19, San Martín, al sur del anterior; San Miguel, al sur del anterior; Egipto-La Peña, comprendido entre las calles 9 y 11 , de la carrera 1 hasta el Paseo Bolívar. En la calle 22 se ubicaba lo que se conocía con el nombre del barrio "Los
Pasajes"; Chiquinquirá, comprendido entre las calles 13 y 15 de la carrera 3 ; otro conglomerado sin nombre, comprendía un conjunto de habitaciones insalubres construidas a lo largo de las calles 4 y 3 al occidente de la carrera 10; Sans Facon, abarcaba de la calle 18 a la 20 , y de la carrera 15 a la 19; Las cruces, situado entre las calles 3 y 2 al sur,

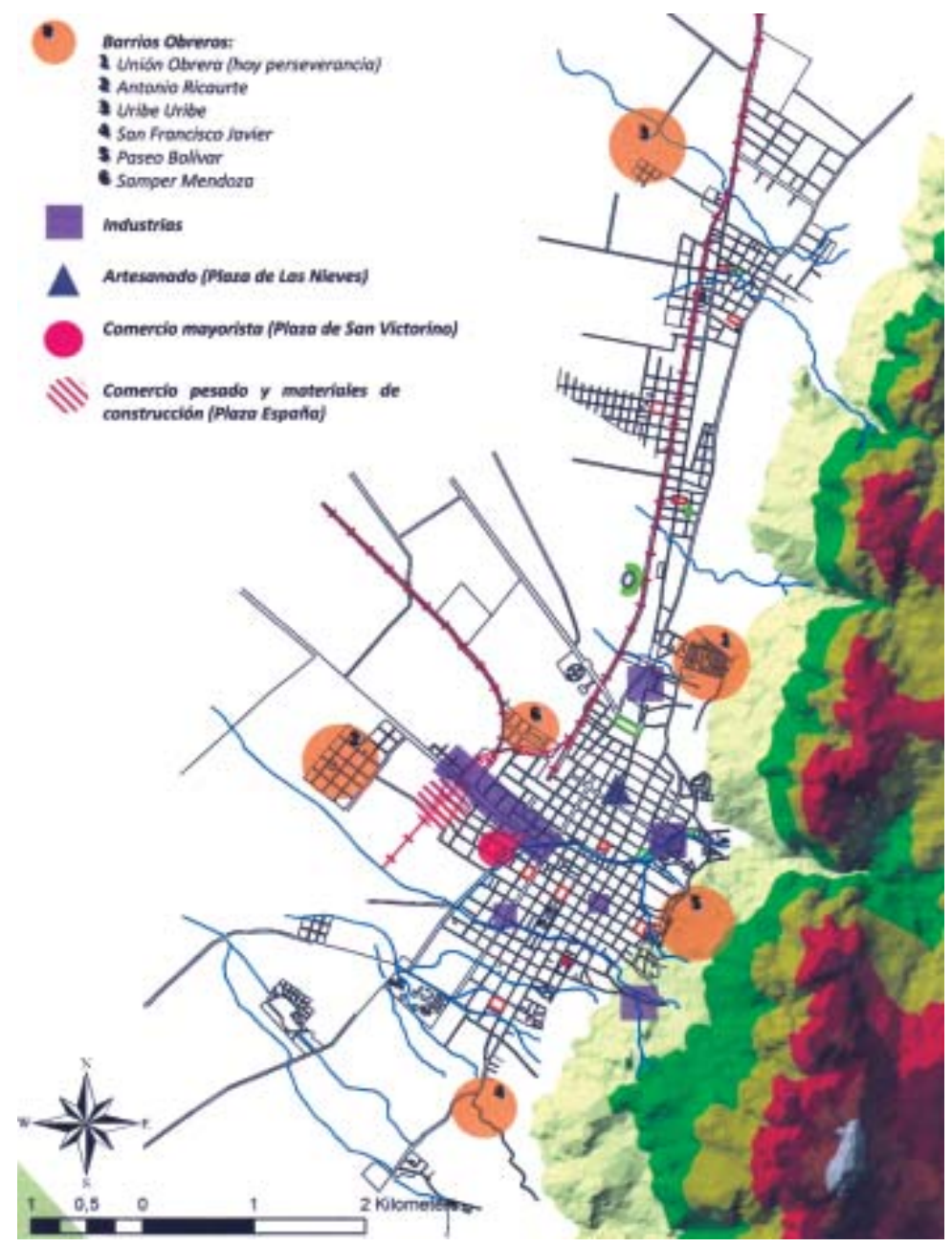

Figura 3. Localización de las principales actividades de la ciudad en 1923

Fuente: Coberturas tomadas de CD Bogotá, Museo de Desarrollo Urbano de Bogotá, SDP. Elaborado por el autor.

$70 \quad$ Luis Gabriel DuQuino Rojas 
de la carrera 6 al oriente. Además había dos núcleos debajo de la carrera 7, al sur de la calle 1. Por último a los lados de los ríos San Francisco y San Agustín, en la parte oriental de la ciudad, se encontraban numerosas casitas pequeñas, de paja, y algunos inquilinatos (Colón, 2007: 108).

\section{Crecimiento poblacional y densificación}

Las poblaciones que se fueron asentando en los nuevos espacios de los barrios obreros bogotanos conformaron el proletariado urbano incipiente; su consolidación y crecimiento demográfico estuvo fundamentado en las nuevas fuentes de empleo industrial y en las constituidas a partir de la inversión estatal en infraestructura urbana y edificaciones para la institucionalidad; estas condiciones se sumaron a las demás razones que impulsaron la migración campo-ciudad (en especial el desplazamiento violento y la escasez de oportunidades y servicios del campo) y que lograran una dinámica de crecimiento poblacional al alza en las dos primeras décadas del siglo $\mathrm{xx}$.

Por otro lado, el flujo de los recursos de la "prosperidad al debe" comenzó a repercutir en los cambios de la dinámica económica, y, a su vez, a través de la inversión pública en infraestructura, en la conformación de una estructura urbana en Bogotá, así: "En 1922 comenzaron a llegar las cuotas de la indemnización de Panamá, y con ellas empréstitos, inversionistas y comerciantes norteamericanos. Entre 1922 y 1928 el país recibió más de 230 millones de dólares, dinero que fue empleado preferentemente en las obras públicas y que incidiera notablemente en este Ministerio -Obras Públicas-" (Niño, 2003). El flujo de estos dineros va a dinamizar el proceso de cambio en curso. La construcción de infraestructura y edificaciones estatales generó nuevas oportunidades de empleo para los migrantes rurales; además, estas fueron constituyendo mejoras implícitas para la actividad industrial y comercial de la ciudad, y aumentaron, con la provisión de servicios públicos, la capacidad urbana para el incremento de las densidades en la ciudad.

Parte importante de la inversión en infraestructura fue a parar a los sistemas de movilidad y transporte:

De hecho, entre 1925 y 1930 se construyó la mitad de la red ferrocarrilera total del país existente en 1930. Por otra parte, entre 1922 y 1934 se duplicó la cantidad de kilómetros de ferrocarriles construidos y en explotación... Además entre 1926 y 1930 se tendieron 942 kilómetros de carreteras y caminos vecinales, de modo que para el último de estos años el país contaba con 2.641 kilómetros de carretera para automotores y 4.042 kilómetros en caminos de herradura, que conectaban regiones como IbaguéArmenia, Pasto-Popayán, BogotáCambao y Villavicencio-Bogotá, además de algunas vías transversales. Ello posibilitó un importante aumento del parque automotor. De hecho, de 552 vehículos importados en 1923, se pasó a 3.556 en 1928, de modo que para 1928 el país disponía de un parque de 
vehículos cercano a las 12.000 unidades, de las cuales un poco más de 2.000 eran de más de 2.5 toneladas (Ocampo, 2007: 221-223).

Estas cifras son indicadores claros del proceso de cambio que vivió el país y que repercutió en su capital directamente, a través de la consolidación de una infraestructura de transporte y de una flota de vehículos de carga que iba sustentando el proceso de crecimiento de los aparatos de producción industriales, en la mejora para el transporte de materias primas y mercancías y en la dinamización de los flujos que conectan un mercado interno, que se robustece, el cual permite abrir oportunidades de negocio a la mercancías de la pequeña y mediana industria floreciente, a nivel regional y nacional. Los procesos de aceleración de la dinámica poblacional, que harán de Bogotá una ciudad mucho más grande y compleja, pues su población casi se triplica en apenas 24 años (figura 4), traerán nuevas problemáticas a un espacio que ya contaba con deficiencias estructurales que, de igual forma, serán amplificadas por las nuevas densidades urbanas.

En el debate público entrarán con fuerza las temáticas de la movilidad, los servicios públicos y los modelos de urbanización por adoptar, temáticas que en años anteriores no tenían la preponderancia que lograrán para esta época, fiel reflejo del tránsito entre un poblado y una ciudad. Algunos de los

\section{Crecimiento de la población en Bogotá entre 1905-1929}

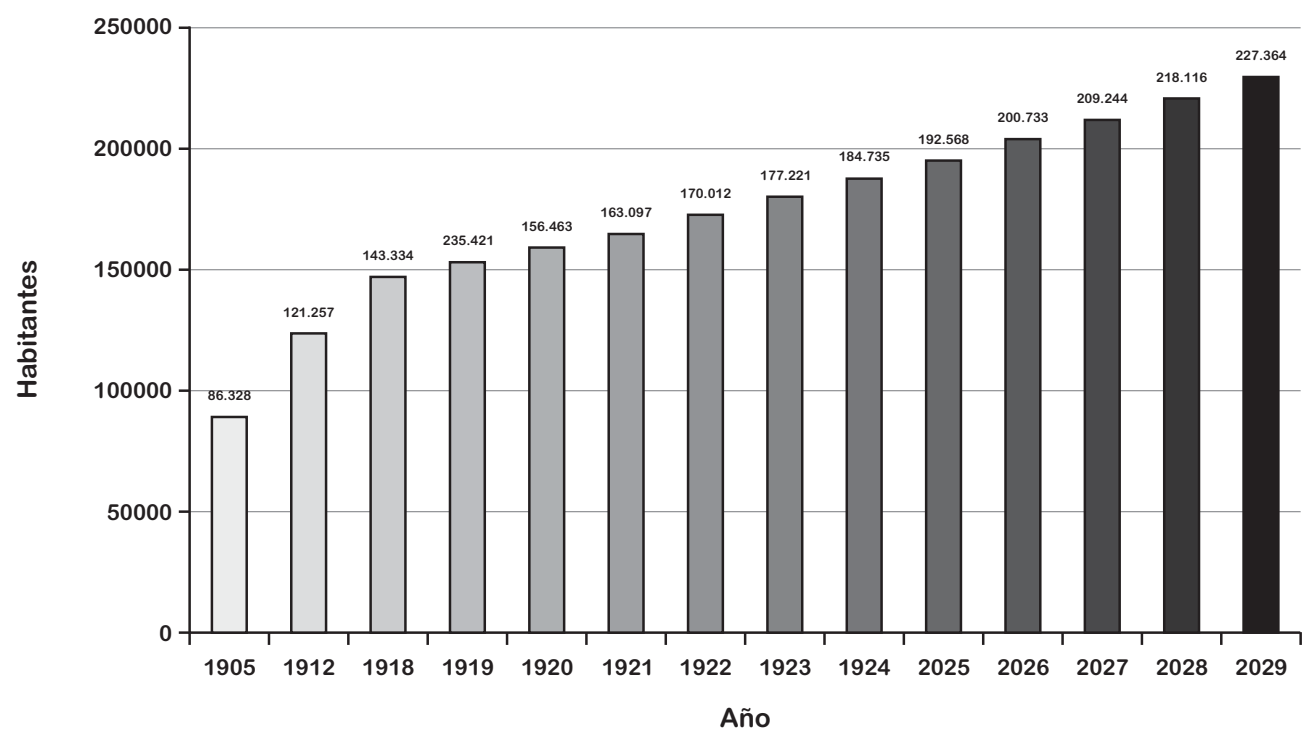

Figura 4. Crecimiento poblacional en Bogotá 1905-1929

Fuente: 1905, tomado de Mejía Pavony, Los años del cambio. Historia Urbana de Bogotá, 1998. Resto, tomado de Suárez, 2006.

72 Luis Gabriel DuQuino Rojas 
primeros emprendimientos para este fin vendrán en principio de la sociedad civil, como lo ejemplifica el caso

del comerciante y dirigente cívico antioqueño Ricardo Olano, y su periódico La ciudad futura. Olano es un pujante hombre de negocios de Medellín, que frecuenta Europa y Norteamérica, se apasiona con el tema de las ciudades modernas e inicia en el país una intensa labor individual, tratando de llamar la atención sobre el arte que rige y reglamenta la creación, crecimiento y conservación de las agrupaciones urbanas, surgido en los últimos años, según su propia expresión (Del Castillo, 2003: 75).

El ejercicio de planificación propuesto desde el principio del debate de la problemática urbanística va a consistir en la importación de modelos foráneos, inicialmente el city planning de Inglaterra, renunciando de entrada a la posibilidad de producir ordenamientos acordes con las dinámicas propias de la realidad socioeconómica nacional. En el proceso de desarrollo de la actividad económica del país y de la institucionalidad construida en paralelo para su gestión, comenzó un proceso de complejización que se va a reflejar marcadamente en el desarrollo urbano de Bogotá y en su papel preponderante como capital de país. Este fenómeno tuvo varias manifestaciones socioeconómicas, entre las que se cuentan: la solidificación de una burocracia con algún grado de tecnificación, germen de las tecnocracias contemporáneas, que bajo sus lógicas de hábitat constituirá nuevos criterios de clase expresados en sus modos de consumo, tanto de mercancías como de espacialidad urbana (mercado inmobiliario) y oferta de entretenimiento. Igualmente, el desarrollo del aparato estatal y financiero generó emprendimientos orientados a la construcción de edificaciones destinadas a estos usos, financiados en el ámbito estatal a través de los flujos de capital financiero de la "prosperidad al debe" y de los dineros de la indemnización del Canal de Panamá.

Las nuevas burguesías liberales, constituidas por los industriales, banqueros, financistas y hasta las élites terratenientes de la Sabana de Bogotá, constituyeron una clase comprometida con la importación de modelos del hábitat urbano europeo y norteamericano, como un escape a las difíciles condiciones materiales presentes en la ciudad, generando los primeros procesos de marginalidad y segregación socioespacial que caracterizarán la ciudad desde su inicial desarrollo; todo esto construido como reflejo de las grandes brechas económicas entre los diversos grupos socioeconómicos.

De acuerdo con el trabajo de Hernando Calvete, la sociedad bogotana estaba compuesta por los tradicionales cachacos, herederos del modelo colonial y representantes de esa burguesía terrateniente de la sabana de Bogotá. A su vez, los guaches hacían parte de la sociedad urbana, y constituían los artesanos de fines del siglo xIx y continuaron en esa tradición hasta bien entrado el siglo $\mathrm{xx}$; estos eran 
carpinteros, peluqueros, zapateros, ebanistas, tejedores, talabarteros, plateros, joyeros y albañiles. En otra categoría estaba el bajo pueblo, que incluía artesanos, vivanderos, comerciantes al menudeo y sirvientes. Un gran número de indígenas que habían sido reducidos por intereses terratenientes de la sabana y que también habían perdido sus tierras comunales engrosaban ahora esta clase social que hacia los trabajos más difíciles en el escenario urbano, en condiciones sumamente adversas... Finalmente, se encuentran los cargueros, peones y mozos de cuerda, en la escala más baja (Preciado et al., 2005: 84).

Este nuevo panorama social fue construyendo una espacialidad propia, en donde se manifestarían con fuerza las brechas sociales existentes entre los grupos que tenían asiento en la ciudad, predefiniendo la marginalidad socioespacial que caracteriza la ciudad hasta nuestros días.

\section{Forma urbana}

En términos morfológicos, Bogotá comienza el siglo xx básicamente ocupando la misma estructura de la ciudad colonial. En buena medida, los procesos de expansión urbana no se dieron tempranamente ${ }^{5}$ por la escasez de recursos para incorporar más suelo a la estructura urbana existente y por los procesos de privatización de los ejidos de la ciudad; esto último debido a la fuerte presión privatizadora $\mathrm{y}$ expansionista de las haciendas en la Sabana de Bogotá (Zambrano, 2007). No solo la estructura urbana mantendrá un fuerte carácter decimonónico, además, las formas de administración del territorio estarán cargadas de las lógicas del siglo XIX, manifestadas en la importancia de la división por parroquias para la organización de la ciudad, propia del modelo agrario/rural y reflejo de la influencia jerárquica del clero en los asuntos civiles.

Por tanto, la organización de la ciudad y sus formas de uso giraban en torno a la vida urbana, que se desarrollaba sobre las plazas y plazoletas de las principales iglesias, que constituían a su vez las parroquias en que estaba divida Bogotá para la época (figura 5). Es así como cada uno de estos binomios (iglesia/plazoleta) se instituyó como una pequeña aglomeración de ciertas actividades, de acuerdo con el papel funcional de la zona para la ciudad. La principal parroquia, La Catedral, con la Plaza de Mayor ${ }^{6}$, aglomeró históricamente las sedes del poder gubernamental y eclesiástico, así como los principales comercios y la vivienda de la élite. De igual forma, este espacio se convirtió en elemento fundamental para la vida urbana, tal como lo narra Sánchez:

En la Bogotá finisecular había un espacio donde se realizaban las

Las expansiones más importantes se darán a partir de la década de los treinta.

6 Actual Plaza de Bolívar.

74 Luis Gabriel DuQuino Rojas 
grandes confrontaciones entre la noticia oficial y el rumor de la oposición: el Altozano. En el puro centro de la ciudad, entre la Catedral y la Plaza, el Altozano tenía cien metros de longitud, y allí, caminando ida y vuelta o formando corrillos, se daban citas las lenguas más vivas e ingeniosas para comentar lo que el gobierno decía o no decía, pero también para desollar al vecino, al rival (Sánchez, 1998).

La plazuela de Las Nieves, la segunda en importancia, alojaba en su entorno la vivienda de los artesanos, gremio considerado para la época como la clase media de la ciudad, en el cual germinaron algunas de las iniciativas de la industria manufacturera naciente en la Bogotá de los veinte y los treinta. Al occidente, como un elemento de relevancia, tenía asiento la parroquia de San Victorino, cuya plazuela, inicialmente llamada de Nariño (posteriormente San Victorino), se ubicaba un conglomerado comercial de relevancia establecido como puerto terrestre, por ser puerta de entrada a Bogotá por el camino a Honda, y por haber recibido, en 1861 (Mejía, 1998), el traslado del mercado que tradicionalmente se asentaba en la plaza de Bolívar. "Asimismo, por su ubicación, ésta se convirtió en el apostadero principal de los omnibuses y carruajes de alquiler que tuvo la ciudad" (Mejía, 1998: 187). En estos tres sectores de la ciudad se podía observar ya la caracterización funcional de la forma urbana desarrollada hasta el momento. El centro de la ciudad, compuesto por las manzanas de la estructura urbana colonial, contenía los espacios más relevantes en cuanto a la actividad económica de la ciudad, hecho reflejado en la concentración de establecimientos institucionales, de comercio y servicios: la totalidad de los bancos, todos los pasajes comerciales, la mitad de los colegios, todos los establecimientos de educación superior, 14 de las 53 fábricas de la ciudad y 16 de los 21 hoteles (Zambrano, 2007). Dicha actividad económica se complementaba con la presencia sobre el eje de la Avenida Colón (calle 13) de actividades comerciales e industriales que se iban consolidando por el papel de este corredor vial como conector de la salida más importante de la ciudad hacia el río Magdalena y de allí a los principales puertos del país.

Más allá de estos espacios que daban cuenta de los límites de la ciudad colonial, se encontraban otros con características similares, los cuales fueron adicionados en los lentos procesos de expansión del casco urbano a principios del siglo xx. En los límites occidentales de la ciudad se encontraba la plaza de Maderas, posteriormente rebautizada Plaza España, que por ser puerta de entrada occidental de la ciudad alojó el comercio pesado de la época, la venta de materiales de construcción y de alimentos al por mayor, en especial la miel (Mejía, 1998). En el límite norte de la ciudad se encontraba el suburbio de Chapinero, que lentamente iba siendo alcanzado por la parcelación que se daba sobre los predios con frente a las carreras 7 y 13; como los sectores anteriormente 
descritos, contaba con la presencia de su conjunto iglesia/plaza; la plaza, desde su origen, se caracterizó por ser un enclave comercial, y en los alrededores se asentó un área residencial de clase alta, con la presencia de grandes casaquintas y estancias que en parte debían su amplio tamaño a los costos reducidos de la tierra en este sector, en comparación con las áreas centrales, que ocupaban las clases más favorecidas.

Toda la actividad institucional, comercial y de servicios concentrada en la zona más central de la ciudad se complementaba con áreas residenciales también definidas como parroquias, y que en el tránsito de la transformación administrativa de la ciudad en la segunda década del siglo $\mathrm{xx}$ se convertirían en barrios (figura 6). Como se estableció anteriormente, la ciudad tenía serias dificultades para posibilitar su expansión; esto generó, entre otras cosas, la concentración de la actividad constructora en los solares desocupados de las áreas existentes, que incluían la vieja estructura de la ciudad colonial con algunas adiciones en sus bordes. La oferta de espacio urbano para construir se complementaba con operaciones de demolición y subdivisión de predios, así como con procesos de reedificación de las casas existentes, de construcción de los patios y subdivisión de las edificaciones construidas desde la Colonia.

En términos urbanos, el hecho más significativo es la densificación del parque inmobiliario y el establecimiento de nuevos patrones de loteo. El solar colonial -un cuarto o sexto de manzanaobedecía a una casa pensada para una familia extensa, numerosa servidumbre y amplios jardines y huertos; en este momento ese tipo de construcciones eran ya obsoletas, pero también lo era el terreno mismo; se va a producir entonces un amplio proceso de subdivisión de lotes tradicionales, dando lugar a un nuevo tipo de lote más pequeño y de proporción alargada (1:3 ó 1:4); el mecanismo normal fue el de comprar una antigua casa, para demolerla y luego subdividir el terreno en 4 ó 5 franjas, produciendo una renovación urbana espontánea y emprendida por particulares, que adquirió grandes proporciones (Arango, 1993: 140).

La imposibilidad de expansión y la actividad edificatoria concentrada en la estructura de la ciudad vieja tuvo duras consecuencias sobre la calidad de vida de los residentes, pues al llevar a cabo estas intervenciones se minaron varias de las condiciones de habitabilidad presentes, relacionadas con la ventilación, la iluminación y la disposición de los pocos servicios con que contaba la vivienda hasta ese momento. Los primeros debates sobre la problemática de la reedificación se dieron desde un movimiento que ganaba fuerza en el mundo a principios del siglo $\mathrm{xx}$, constituido por los profesionales del sector de la salud: el higienismo. El discurso del higienismo se afianzó tras eventos epidémicos que se dieron por las deficientes condiciones de salubridad en la ciudad, las cuales se agravaban permanentemente como fruto de los

76 Luis Gabriel DuQuino Rojas 
procesos de crecimiento poblacional y densificación de la estructura urbana; la de gripe de 1918 es un buen ejemplo, dejó más de 1.200 muertos (Sánchez, 1998). Varios de los planteamientos de los higienistas de la época se caracterizaron por brindar una lectura muy acertada de la situación de transformación urbana presente en la época; por ejemplo, el doctor Manuel Lobo, que en el periódico El Tiempo, del 27 de mayo de 1914, planteaba lo siguiente:

Los habitantes de las ciudades necesitan amplios espacios para respirar aire puro. En este sentido las condiciones de Bogotá han desmejorado mucho en los últimos tiempos. Las habitaciones construidas en la época de la colonia y en los primeros lustros de la vida independiente, aunque un poco bajas de techo y provistas de anchos corredores que impedían la entrada de sol a las piezas, tenían varios patios grandes con jardines y solares donde abundaban los árboles. Esto estaba de acuerdo con las costumbres conventuales de la época. Poco importaba a los moradores de entonces que las calles fueran estrechas y tortuosas y que no hubiera plazas y parques; dentro de su casa tenían donde pasearse y darse baños de sol. Además las salidas al campo eran muy frecuentes. Con el aumento de población

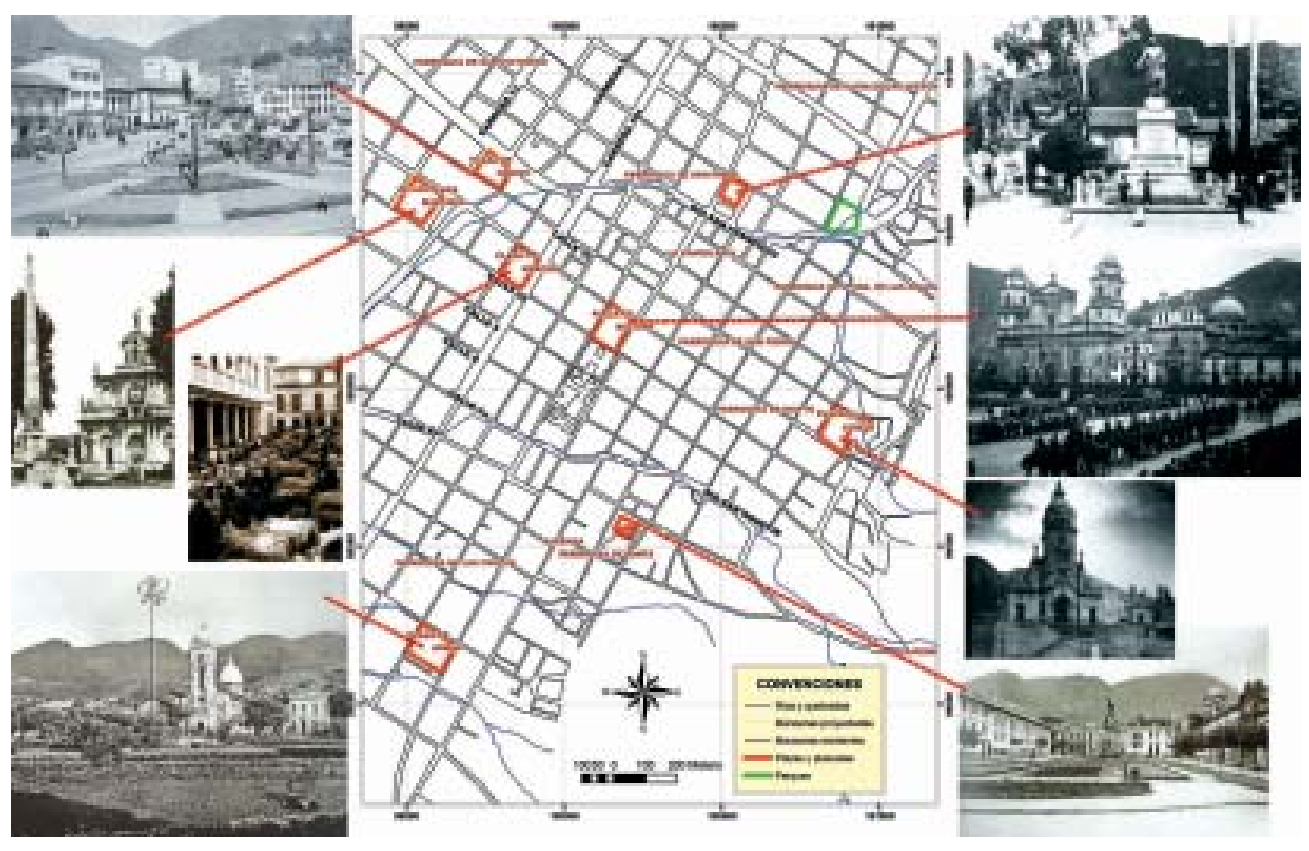

Figura 5. Plazas, plazuelas y parroquias, Bogotá 1923

Fuente: Coberturas tomadas de CD Bogotá, Museo de Desarrollo Urbano de Bogotá, SDP. Realizado por el autor. 
esas antiguas casas van modificándose y desapareciendo. De una casa antigua se hacen otras dos modernas, reduciendo los patios y suprimiendo los solares. Entre tanto que las casas estrechas en su interior, las angostas calles españolas continúan con sus antiguas dimensiones y las plazas y parques no aumentan en la proporción que debieran.

La situación fue configurando una problemática que se presentaría persistentemente en la evolución urbana de la ciudad; los cambios estructurales que se fueron dando en la composición social, en la economía y en los modos de habitar, no se acompañaron de repercusiones profundas en la forma urbana, lo que trajo una serie de dificultades que se acrecentarán en la medida en que la ciudad se densificaba y crecía.

Los sectores residenciales que complementaban las áreas centrales de comercio y servicio para la época están representados por la parroquia de Santa Bárbara, al sur de la catedral, un sector de vivienda para clases acomodadas; la parroquia de Egipto, en el borde oriental de la ciudad, que alojaba sectores populares; Las Cruces, localizada al sur de Santa Bárbara, habitado por artesanos y clases trabajadoras; la ocupación del Paseo Bolívar, un sector marginal en el costado oriental de la ciudad, que habitaban las clases más empobrecidas, y el barrio obrero de La Perseverancia, en donde tenían asiento las emergentes clases proletarias, principalmente los obreros de la Cervecería Bavaria y de la fábrica de vidrios Fenicia.

\section{Transporte público, tranvía}

En términos de configuración espacial se puede observar que la ciudad de la década de los veinte tenía una disposición lineal, a manera tentacular, sobre los ejes de conexión vial, y jalonada, con anterioridad, por los desarrollos de la red del tranvía, elemento fundamental en los procesos expansivos que se consolidarían en las décadas siguientes.

La línea del tranvía que comunicaba el centro de la ciudad con el suburbio norte de Chapinero, construida en los años 1883-1884 (Martínez, 1983), dinamizó el posterior crecimiento de la ciudad en este sentido. La línea tenía origen en la Plaza Santander y discurría a través de la carrera $7^{\mathrm{a}}$ hasta llegar a San Diego, de allí en adelante continuaba por el camino "nuevo", actual carrera 13 , sobre el cual había muy poca actividad para la época. De igual forma, Chapinero contó con la conexión ofrecida por el ferrocarril del norte, que tenía punto de partida en la carrera 13 con calle 18 y se desarrollaba por donde hoy discurre la Avenida Caracas y la Avenida Paseo de los Libertadores (Martínez, 1983). La disposición de esta conexión vial cumplió un papel fundamental en la expansión de la ciudad hacia el norte, generando una ocupación progresiva de las áreas que separaban el casco urbano colonial del suburbio de Chapinero, las

78 Luis Gabriel DuQuino Rojas 


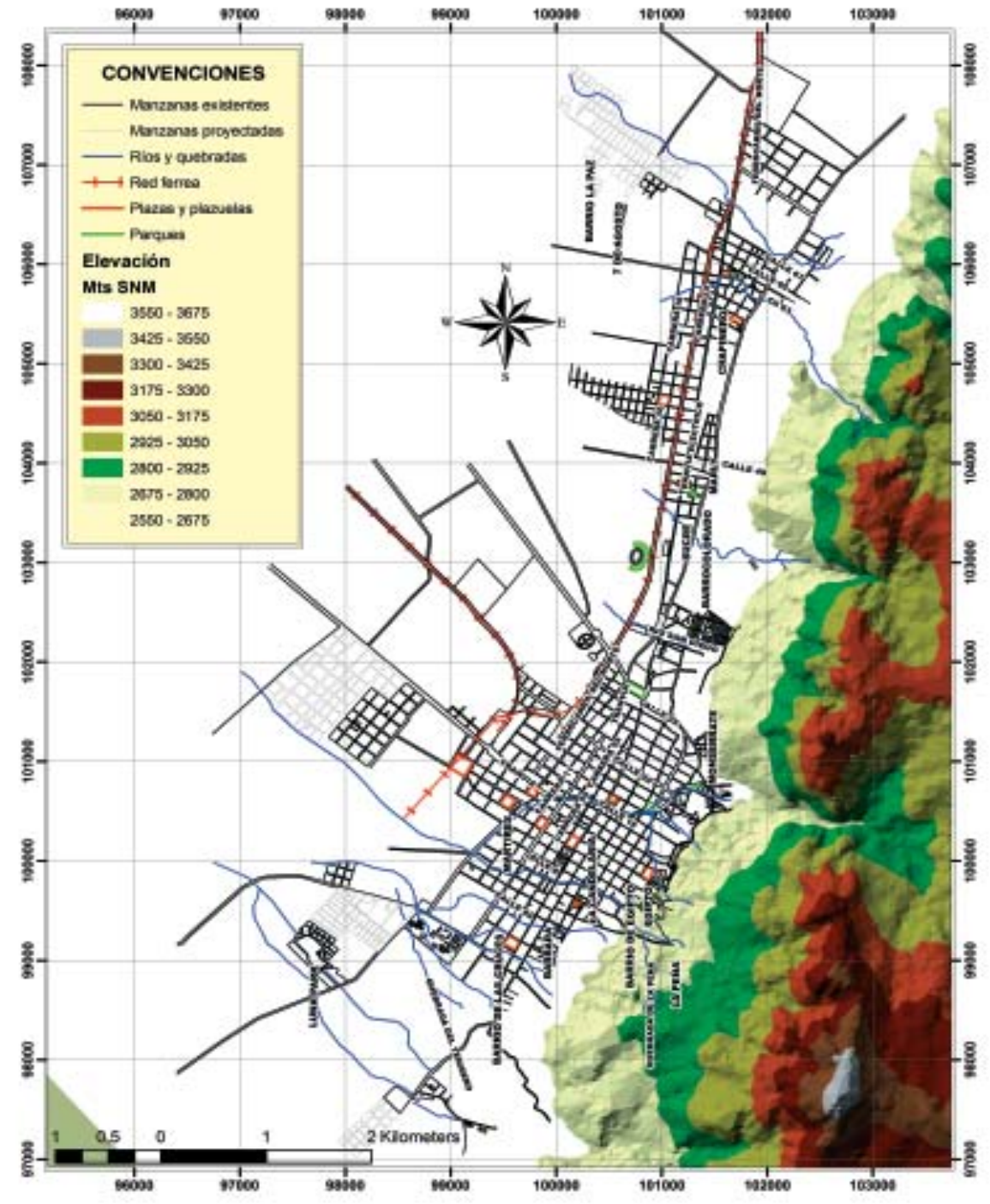

Figura 6. Bogotá para 1923

Fuente: Coberturas tomadas de CD Bogotá, Museo de Desarrollo Urbano de Bogotá, SDP. Elaborado por el autor.

cuales no contaban con una sólida ocupación en épocas anteriores; esta situación se va a manifestar en los aumentos poblacionales de este sector norte y en que el eje centro-norte ocupara un papel de total relevancia en el crecimiento de la actividad urbanizadora de la época. 
De forma similar, la disposición de la línea del tranvía que comunicaba el casco central con la periferia sur (figura 7), en primera instancia hasta Las Cruces, cobrará importancia en la continuidad del desarrollo urbano en el eje norte-sur. La eficiencia y celeridad en la construcción de la línea del norte contrasta con la ausencia de un emprendimiento real por mejorar la conexión hacia el sur, en especial la necesaria conexión con el barrio de San Cristóbal, que nunca se construirá; los intentos por construir esta línea van a quedar en manos de inversionistas privados, que fundaron la Compañía del Tranvía de San Cristóbal, con el objetivo de valorizar terrenos que tenían sobre este sector, en ausencia de un interés estatal por exigir a la Bogotá City Railway Company que amplíe su servicio hacia esas áreas de la ciudad. Esto se convirtió en un reflejo más de la naciente segregación socioeconómica, a favor de los intereses de las clases acomodadas tradicionales, que tenían la propiedad de los terrenos al norte de la ciudad y que valorizaron ampliamente sus tierras a través de las redes férreas (tranvía y tren) tendidas hacia el norte. "La introducción del ferrocarril urbano en el eje sur-norte valorizó esta parte de la ciudad, en detrimento del resto centro, sur y occidente-, lo cual contribuyó a amplificar el desequilibrio socioeconómico y espacial -entre el norte y el sur de la ciudad-; así mismo, dio paso a una transformación en la forma de segregación" (Montesuma, 2008: 96).

Hacia el occidente, la línea del tranvía llegaba a la Estación de la Sabana (figura 7), lugar que tenía un papel funcional de puerta de entrada de la ciudad tanto para viajeros nacionales como internacionales, que arribaban a través el camino a Honda, la salida al río Magdalena. Por tanto, esta conexión del tranvía hacia occidente se caracterizó por ser un intercambiador modal entre el transporte regional y nacional y el propiamente urbano. Los predios aledaños a la Estación de la Sabana fueron destinados al comercio pesado, como se estableció con anterioridad. En conclusión, las más importantes tendencias de expansión y urbanización de la ciudad en los sentidos norte, sur y centro-occidental estuvieron acompañadas de la aparición del tranvía como elemento catalizador de dichas dinámicas, convirtiéndose además en un sistema por el cual se apalancaron algunos de los procesos de valorización de la tierra, los cuales trajeron consigo igualmente los primeros procesos de segregación socioeconómica y espacial.

El proceso anteriormente descrito puede verificarse al revisar las tendencias de crecimiento poblacional de los diversos sectores que componían la ciudad. En la Tabla 1 aparecen relacionados los porcentajes de mayores crecimientos de población, los 


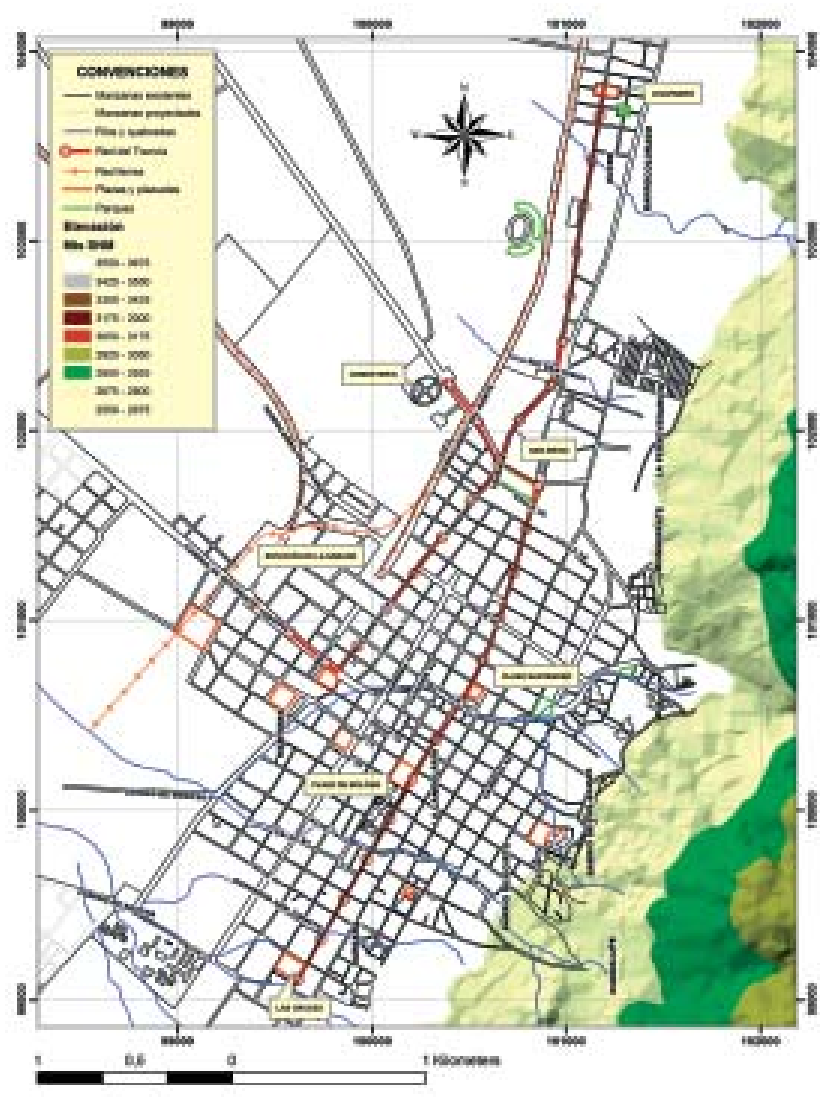

Figura 7. Red del Tranvía en 1910

Fuente: Coberturas, CD Bogotá, Museo de Desarrollo Urbano de Bogotá y SDP. Elaborado por el autor.

Tabla 1. Dinámica poblacional entre 1912-1919

\begin{tabular}{|l|l|c|c|c|c|c|c|}
\hline \multicolumn{1}{|c|}{ Sector } & \multicolumn{1}{|c|}{ Barrio } & $\begin{array}{c}\text { Población } \\
1912\end{array}$ & Porcentaje & $\begin{array}{c}\text { Población } \\
1918\end{array}$ & Porcentaje & $\begin{array}{c}\text { Evolución } \\
\text { 1912-1918 } \\
\text { (en porcentaje) }\end{array}$ & $\begin{array}{c}\text { Tasa de crecimiento } \\
\text { anual 1912-1919 } \\
\text { (en porcentaje) }\end{array}$ \\
\hline Centro & Santa Bárbara & 21.547 & 17.8 & 16.073 & 11.1 & -25 & -5 \\
\hline Norte & San Diego & 12.612 & 10.4 & 8.430 & 5.8 & -33 & -6 \\
\hline Sur & Las Cruces & 14.638 & 12.1 & 19.990 & 13.8 & 37 & 5 \\
\hline Suroriente & Egipto & 15.572 & 12.8 & 17.366 & 12.0 & 12 & 2 \\
\hline Oriente & LasAguas & 11.854 & 9.8 & 16.158 & 11.1 & 36 & -2 \\
\hline Centro & La Catedral & 6.759 & 5.6 & 6.129 & 4.2 & -9 & 4 \\
\hline Occidente & San Victorino & 14.004 & 11.5 & 17.720 & 12.2 & 27 & 3 \\
\hline Centro norte & Las Nieves & 12.735 & 10.5 & 15.125 & 10.4 & 19 & 4 \\
\hline Norte & Chapinero & 7.236 & 6.0 & 9.277 & 6.4 & 28 & - \\
\hline Suroriente & San Cristóbal* & - & 0.0 & 6.292 & 4.3 & - & - \\
\hline Norte & Sucre* & - & 0.0 & 7.374 & 5.1 & - & 3 \\
\hline Sur & Nazaret & 4300 & 3.5 & 5.092 & 3.5 & 18 & 3 \\
\hline
\end{tabular}

Fuente: Tomado de Montesuma, Ricardo, La ciudad del tranvía, 1880-1910, Universidad del Rosario, 2008, p. 94.

Perspect. geogr. Vol. 15. Año 2010, pp. 61-84 181 
cuales se localizan en los sectores norte (San Diego y Chapinero), sur (Cruces) y occidental (San Victorino), acompañados del desarrollo de la red del tranvía.

En la década de los veinte, Bogotá comenzó a expresar espacialmente con mayor intensidad el proceso de transformación del modelo agrícola/ rural hacia el modelo industrial/urbano que se desarrollaba en el país, a través del crecimiento de la población urbana, el surgimiento de un incipiente proletariado -asalariado y en condiciones de mayor libertad que el campesinado-, la complejización de la institucionalidad para el gobierno de la ciudad y la aparición de nuevos usos del suelo. En comparación con la ciudad construida en la actualidad, para el final de los años veinte, el territorio ocupado por Bogotá apenas llegaba a las 1.300 ha, que representan el 3,38\% de la extensión del suelo urbano hoy, correspondiente a 38.430 ha, y la población estaba estimada en 227.364 habitantes, que representan poco más del 3\% de la población actual de la ciudad: 7.363 .782 habitantes.

\section{Conclusiones}

El proceso de evolución de la espacialidad de Bogotá en la década de los veinte se construyó sobre las intensas transformaciones que en la sociedad colombiana se fueron consolidando en este periodo. El territorio de la ciudad es una expresión espacial de los acomodamientos propios de un proceso de evolución de las Formas Económicas Sociales (FES), que de acuerdo con la jerarquía de los intereses de clase de los individuos que componen dichas FES se van imponiendo en mayor o menor grado. Las ideologías, como perspectivas desde las cuales la sociedad interactúa sobre el mundo, corresponden a la supraestructura, que orienta los procesos de relacionamiento por medio de los que se constituyen las FES, definiendo el papel de cada clase y de cada individuo en la estructura jerárquica social. El sistema capitalista, como modo de producción presente en todo el periodo histórico estudiado, fue manifestando cambios orientados desde los espacios de poder del capital mundial, en la búsqueda de nuevas, mejores y más eficientes formas de acumulación económica. Dichos cambios en la evolución del modo de producción se manifiestan en fases que tuvieron énfasis de acuerdo con la actividad económica más intensamente desarrollada: comercial, industrial, financiera y de servicios ${ }^{7}$. La economía colombiana, desde siempre inserta en

Dichas fases se refieren al acento y preponderancia de la actividad productiva que en cada momento histórico se manifiesta, teniendo presente que en cada fase las actividades de explotación anteriores siguen presentándose, pero con un rol diferente dentro de la economía del territorio estudiado.

82 Luis Gabriel DuQuino Rojas 
el contexto de la economía mundial (como espacio de explotación), recibe la orientación de los cambios propuestos desde los centros de poder económico mundial y asume su rol en la división internacional de la producción y el trabajo, generando acomodamientos internos tendientes a organizar la estructura social, política, económica y espacial, con el fin de lograr ejecutar el papel asignado con el mayor grado de eficiencia posible.
Los cambios en la espacialidad de Bogotá, que para principios del siglo xx no pasaba de ser un pueblo grande, se fueron manifestando en la complejización de las estructuras sociales y las actividades que se desarrollaban en el territorio, expresándose en el proceso de tránsito hacia una realidad urbana característica de las sociedades inscritas en la fase del capitalismo industrial.

\section{Literatura citada}

Amézquita, A. (2007). Áreas residenciales en Bogotá. Barrios obreros bogotanos. Urbanismos, 2: 93-103.

Arango, S. (1993). Historia de la arquitectura en Colombia. Bogotá: Universidad Nacional de Colombia.

Colón, L. C. (2007). El saneamiento del Paseo Bolívar y la vivienda obrera en Bogotá. Urbanismos, 2: 104-115.

Del Castillo, J. C. (2003). Bogotá. El tránsito a la ciudad moderna 1920-1950. Bogotá: Universidad Nacional de Colombia.

Harvey, D. (2003). Espacios de esperanza. Madrid: Akal. Leff, E. 2002. Saber ambiental. Sustentabilidad, racionalidad, complejidad y poder. México: Siglo XxI.

Martínez, C. (1978). Bogotá reseñada por cronistas y viajeros ilustres. Bogotá: Escala.

Martínez, C. (1983). Bogotá, sinopsis sobre su evolución urbana. 1536-1900. Bogotá: Escala.

Mejía, G. (1998). Los años del cambio. Historia urbana de Bogotá 1810-1910. Bogotá: Pontificia Universidad Javeriana. 
Montezuma, R. (2008). La ciudad del tranvía 1880-1920. Bogotá: Transformaciones urbanas y movilidad. Bogotá: Universidad del Rosario.

Niño, C. (2003). Arquitectura y Estado. Bogotá: Universidad Nacional de Colombia.

Ocampo, J. A. (Compilador). (2007). Historia económica de Colombia. Bogotá: Planeta.

Preciado, J. et al. (2005). Historia ambiental de Bogotá, siglo xx: elementos históricos para la formulación del medio ambiente urbano. Bogotá: Fondo de Publicaciones Universidad Distrital Francisco José de Caldas.

Sánchez, C. (1998). De la aldea a la metrópoli. Seis décadas de vida cotidiana en Bogotá, 1900-1959. Bogotá: Instituto Distrital de Cultura y Turismo, Tercer Mundo.

Zambrano, F. (2007). Historia de Bogotá. Siglo xx. Bogotá: Villegas Editores. 